Original Research Paper

\title{
Multilevel Mixed Analysis and Mapping on Divorce Problem in UAE
}

\author{
Faisal G. Khamis \\ College of Business Administration, Al-Ain University of Science and Technology, Al Ain, UAE
}

Article history

Received: 22-06-2016

Revised: 30-09-2016

Accepted: 26-10-2016

Email: faisal_alshamari@yahoo.com

\begin{abstract}
Despite the wealth of research investigating the divorce problem in the developed countries, few and inconsistent studies investigated this problem at emirate-level in developing countries, such as United Arab Emirates (UAE). The Questions are raised whether divorce is changed over time and whether clustering in divorce exists within emirates in UAE. The objectives are to investigate the change in divorce over time, examining the variation between- and within emirates, determining whether the clustering in divorce within-emirates exists and forecasting divorce in each emirate for the next years. The study design was cross-sectional time-series data. Multilevel mixed-effects linear regression was carried out. The data of 7 emirates over 19 years (1995-2013) were obtained from the National Bureau of Statistics. After calculating the Divorce Rate (DR), visual inspection for the DR was investigated using mapping. Three multilevel models for the DR were estimated and comparison between these models was explained in Intra-Class Correlation (ICC), the proportional change in the variance of the DR and the deviance. The $p<0.01$ of Wald- $\chi^{2}$ was found significant in all models. Given $95 \%$ confidence interval, the fixedand random-effects in all models were found significant. The ICC results were found significant, more than $46 \%$, in all models. In terms of the statistical and social epidemiological concepts of contextual phenomena confirm that the DR from the same emirate is more similar to each other than those from different emirates. Using the time as a predictor for the DR reduced slightly the within-emirates variance and inflated dramatically the between-emirates variance.
\end{abstract}

Keywords: Multilevel Modeling, Divorce, Time, Emirate, Forecasting and Intra-Class Correlation

\section{Introduction}

The multilevel mixed-effects linear regression model is used whenever the data is grouped in more than one category, for example, the emirates in the current study. However, using multilevel modeling allows researchers to investigate simultaneously the effect of individuallevel and group-level predictors on the response variable of interest. Multilevel model is a hierarchical model that contains variables measured at different hierarchical levels. Due to multilevel model acknowledges hierarchical data, researchers should not move to a single model in their studying aggregated or disaggregated variables (Hox, 1995). Thus, there are statistical and conceptual advantages in choosing.
In the present study, the importance of the context was highlighted to explain that the social differences may differ over time with different characteristics. So, time series data were obtained for the divorce variable across 7 emirates in UAE and over the time period (1995-2013) of 19 years. Time series data allows controlling for variables which cannot measure like cultural factors or difference in business practices across companies; or variables that change over time but not across emirates (i.e., national policies, federal regulations, international agreements, country rules and legislations.). These variables account for emirates heterogeneity.

The objectives of the current study are: Investigating the variation in divorce in UAE over time, measured by years and across space, measured by emirates. 
Identifying whether the clustering in divorce exists in UAE using Intra Class Correlation (ICC) among observations within the same emirate. Introducing Multilevel Regression Modeling (MRM) in a different way and in an understood, easy and simple statistical details for the statisticians and non-statisticians, compared with many previous studies which applied the MRM, to be used by many researchers in their studies. This is due to several advantages involved in the technique of MRM, such as, the dependency among observations that is not allowed by familiar regression modeling. Introducing visual inspection for the DR using mapping.

Several questions could be asked in the present study. What would be the average of the 7 regression equations for 7 emirates? i.e., what would be the average of the intercept and the slope of these equations? How much variation in the DR can be explained by the time? How the differences in the DRs are partitioned in a variability that exists between the rates from the same emirate and a variability that exists between emirates.

The divorce problem was investigated by many researchers around the world. The UAE has recorded the highest the DR in the region, where the figure was more alarming for Emiratis, who make up less than one-fifth of the population but account for over one-third of all divorces (Express, 2013). Webster (2015) stated that financial pressures, poor communication and the rampant rise of social media have all been blamed for the UAE's rising divorce rates and the DR may be comparable to that in Europe but the number of couples choosing to split in the UAE is increasing at a faster rate, according to some experts. Mednick (1987) explored the long-term effects of parental divorce on young adult male crime from a longitudinal perspective in Denmark. They found a significant relationship between divorce and young adult crime. The evidence suggested that the negative relationship between women's education and divorce is weaker when marriages involve abuse than when they do not (Kreager et al., 2013). Bourne et al. (2014) found that the DR is positively correlated with Murder Rate (MR), in Jamaica, with the DR accounting for most of the variance in the MR. They used ordinary least squares regression analysis and the time period of their study was (1950-2013). In Oklahoma, Bishop et al. (2015) examined differences in self-reported dispositional forgiveness types among older male prison inmates who experienced parental divorce. They found significant mean differences for the forgiveness of situation. In the USA, Furtado et al. (2013) indicated that divorce culture has a stronger impact on the divorce decisions of females than of males, pointing to a potentially gendered nature of divorce taboos using regression analysis. They used data from the immigrants from Europe who arrived in the USA when they were age 5 or younger.
The current study is very important due to several reasons. First, increasing the DRs even with small rates in any community has dramatically bad effects on the overall children life and on the mental health of all family members. Children of divorced parents often have social problems. They may lack support or care in the home or have difficulties in the new family and they have problems at school. Second, as a consequence on the long-run, the divorce will destroy the community as a hall due to building a strong family is the basis stone for having a concrete and healthy community. Third, to the author's best knowledge no study has investigated the DR over relatively a long period of time and across all emirates in UAE. Fourth, there is an abundance of social problems, such as divorce, in developing countries that affect long-term health conditions and could contribute to health inequalities. Fifth, the high levels of divorce have had many effects on the economy.

The contents of the current study include five sections. Section one shows the introduction which introduces the problem, why it is important, how does the study relate to previous work in the area, what are the hypotheses and objectives of the study and what are the theoretical and practical implications of the study? Section two shows the data used in the analysis and explains the steps of analysis with some statistical details. Section three includes detailed results showed in figures and tables. The discussion is explained in section four with some details regarding the support of the study hypotheses, why the MRM technique is applied in the current study and how much the findings in the recent study are related to the findings in the previous studies. Conclusions and recommendations are explained in section five.

\section{Materials and Methods}

\section{Data}

The UAE was selected because of its importance in the gulf region and the good quality data. Historically, in UAE, the DR decreased in most emirates over the time period under investigation. The data for nationals on 7 emirates were obtained from the National Bureau of Statistics. The rate of the divorce for the $i$ th emirate was calculated as follows:

$$
\text { rate }_{i}=\left(O_{i} / n_{i}\right) 1000, \quad i=1,2, \ldots, 7
$$

Where:

$O_{i}=$ An observed number of divorce

$n_{i}=$ The population size of the $i$ th emirate

\section{Analysis}

The measures of variance in the DRs between emirates and Intra-Class Correlation (ICC) within- 
emirate were investigated to understand the distribution of some common social problem in the Emirati population. The research design was a cross-sectional and time series analysis using multilevel mixed regression models. Seven steps of analysis were conducted. In step one the DR was calculated and then was tested for whether it follows a normal distribution using Kolmogorov-Smirnov test. It was found to follow approximately a normal distribution. Step two involved descriptive statistics for the DR across emirates and over time. In step three, three models of multilevel mixed models for DR were estimated using Stata Software. In step four, the ICC, the proportional change in the variance of the $\mathrm{DR}$ between-emirates $\left(\mathrm{PCV}_{\mathrm{BG}}\right)$ and within-emirates $\left(\mathrm{PCV}_{\mathrm{WG}}\right)$ and the deviance were calculated for each model. In step five, a comparison between the estimated models was explained and discussed in some details in terms of ICC, PCV and the deviance. In step six, spatiotemporal mapping was conducted for visual inspection. In step seven, the random-effects were predicted to forecast the DR for the next years.

\section{Multilevel Mixed Regression Analysis}

The Linear Mixed Models (LMMs) procedure expands the general linear model so that the error terms and random effects are permitted to exhibit correlated and non-constant variability. The multilevel model can study the effects that vary by emirates and can estimate emirate level average. The LMMs are used when there is some sort of clustering in the data. Observations from the same cluster are usually more similar to each other than observations from different clusters. If they are, statistical methods can't be used on these data to that assume independence due to estimates of variance and $p$ values will be incorrect. Multilevel models not only account for the correlation among observations in the same cluster, it gives us an estimate of that correlation.

The linear mixed modelling is called multilevel modelling due to the variance and clustering in the response variable can be studied at multiple levels of analysis. So here level-1 and level-2 explain the variation in the DRs within-emirates and between-emirates respectively. Regular regression ignores the average variation between-emirates and individual regression may face sample problems and lack of generalization. The variation across emirates is assumed to be random and uncorrelated with the covariate. Models were fitted using maximum likelihood method. Figure 1 shows research framework which explains the structure of a multilevel proposition.

The details of just one model, which is model-3, out of three models were explained and the details for the other models are same and easier to be known by the readers. So, in the combined model-3, the level-1 model was investigated to examine the variations withinemirates and explains these variations over time. Leve-2 model was investigated to examine the differences between-emirates and explains these differences in terms of emirate characteristics. The combined LMM contains both fixed and random effects, where the LMM for the DR response, affected by the covariate of time, can be shown as follows:

$$
\begin{aligned}
& D R_{i j}=\beta_{0}+\beta_{1} \text { time }_{i j}+u_{0 j}+u_{1 j} \text { time }_{i j}+\varepsilon_{i j} \\
& i=1, \ldots, 19 \text { years and } j=1, \ldots, 7 \text { emirates }
\end{aligned}
$$

Where:

$D R_{i j}=$ Represents an outcome of the DR for the $i$ th year in the $j$ th emirate

$\beta_{0}=$ Represents an intercept term (overall mean intercept of all emirates)

$\beta_{1}=$ Represents a regression coefficient (fixed slope) of time (overall mean slope of all emirates)

$u_{0 j}=$ the random intercept for the $j$ th emirate. It's really a residual term that measures the distance from each emirate's intercept around the overall intercept, $\beta_{0}$. Rather than calculating an estimate for every one of those distances, the model is able to just estimate a single variance, $\sigma_{0}^{2}$

$u_{1 j}=$ The random slope of time for the $j$ th emirate

$\varepsilon_{i j}=$ The overall error term (level-1 errors) of the DR for the $i$ th year in the $j$ th emirate

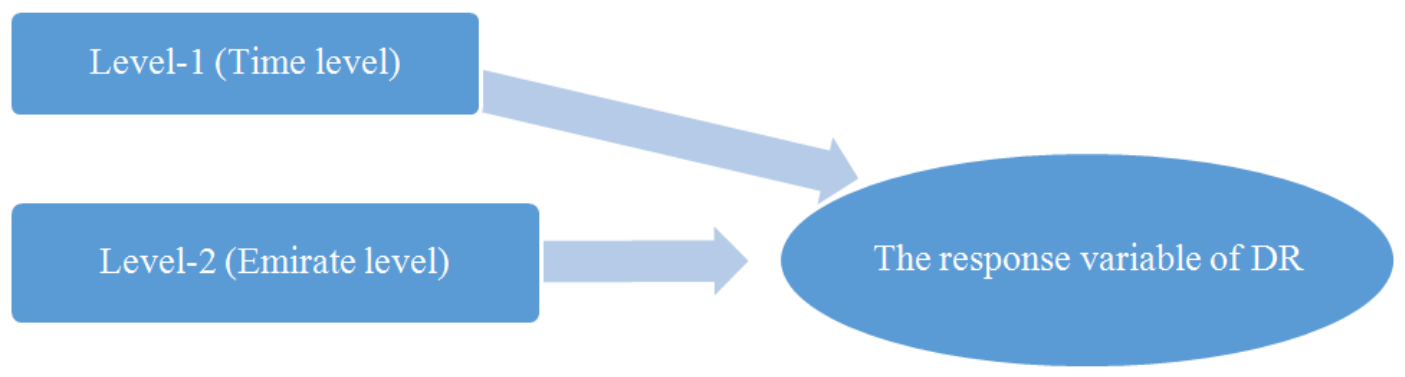

Fig. 1. Research framework shows the structure of a multilevel proposition 
Fixed-effects are used to model averages of regression coefficients whereas random-effects are used to model differences in emirate-variance. The fixed portion of the above model, $\beta_{0}+\beta_{1}$ time $_{i j}$, states that one overall regression line representing the population average is required. Fixed-effects are important for studying the impact of a variable over time. The random effect, $u_{0 j}$, shifts the regression line up or down according to each emirate and the random effect, $u_{1 j} t i m e_{i j}$ explains the effect of time on the DR across the emirates. The random effects occur at the emirate-level (level-2). Random-effects are very important for studying the impact of a variable across emirates. The $u \sim N\left(0, \Sigma_{u}\right)$ independently of $\varepsilon \sim N\left(0, \sigma_{\varepsilon}^{2} I\right)$. The errors $\varepsilon_{i j}$ are assumed to be homoscedastic and not correlated. The within-emirate variance, $\sigma_{\varepsilon}^{2}$, represents how far each $i j$ th observation of the DR is to the emirate specific mean. Covariance structure was not specified for the level-2 $u$ terms due to the covariance between $u_{0 j}$ and $u_{1 j}, \hat{\sigma}_{01}$ wasn't found significant. So, the variance-covariance matrix of $\left(u_{0 j} u_{1 j}\right)^{\prime}$ can be shown as follows:

$$
\Sigma_{u}=\operatorname{var}\left[\begin{array}{l}
u_{0 j} \\
u_{1 j}
\end{array}\right]=\left[\begin{array}{ll}
\sigma_{0}^{2} & \\
& \sigma_{1}^{2}
\end{array}\right]
$$

where, $\sigma_{0}^{2}$ represents the between-emirates variance in the $\mathrm{DR}$, i.e., how far each $j$ th mean of the DR is to the overall emirates mean and $\sigma_{1}^{2}$ represents betweenemirates variance in the DR due to the time.

Table 1 shows three equations of multilevel models under investigation. Model-1 is called empty model which do not include explanatory variables but estimate the emirate Response Variable (RV) mean and the emirate- and individual-levels differences in the RV. In this model, the variation in the RV between-emirates is assumed to be of similar magnitude for each observation. So, by using this model, the aim is to identify a possible contextual phenomenon that can be quantified by clustering of RV within-emirates. Model-1 is expanded to model-2 by including the time variable as fixed-effect and then model- 2 is expanded to model- 3 by including the time variable as fixed- and random-effect.

\section{Intraclass Correlation}

The ICC is a measure of similarity among the DRs over time within the same emirate and therefore, can be used to operationalize the concept of contextual phenomena (Merol, 2003). Individuals who live in the same emirate may be more similar to each other than individuals who live in other emirates, as they share a number of socioeconomic and demographic characteristics that may condition similar social and economic statuses (Merol, 2003). The ICC can be interpreted as the proportion of variance in the DR that is accounted for or explained by clustering. However, the ICC helps us to determine whether or not an LMM is necessary. The ICC can be calculated, for instance, for model-3 as follows (Merol, 2003):

$$
I C C=\frac{V A R_{2 \text { nd. level }}}{V A R_{2 \text { nd. level }}+V A R_{\text {lst. level }}}=\frac{\hat{\sigma}_{0}^{2}+\hat{\sigma}_{1}^{2}}{\hat{\sigma}_{0}^{2}+\hat{\sigma}_{1}^{2}+\hat{\sigma}_{\varepsilon}^{2}}
$$

\section{Proportional Change in Variance}

The proportional change in variance at different levels can be explained as follows: The proportional change in the variance of the DR between-emirates $\left(\mathrm{PCV}_{\mathrm{BE}}\right)$ and within-emirates $\left(\mathrm{PCV}_{\mathrm{WE}}\right)$ explain the variance in the DR between-emirates and withinemirates respectively by differences in the explanatory variables. The differences between emirates in the DR may be attributable to contextual influences or to the differences in the individual composition of emirates in terms of time and other individual characteristics, such as socioeconomic, not considered in the current study. By adjusting for individual characteristics, some part of the compositional differences were taken into account and some of the emirate variance detected in the empty model were explained. The $\mathrm{PCV}_{\mathrm{BE}}$ and $\mathrm{PCV}_{\mathrm{WE}}$ can be calculated respectively as follows (Merlo et al., 2005b):

$$
\begin{aligned}
& \mathrm{PCV}_{\mathrm{BE}}=\frac{\hat{\sigma}_{0}^{2} \text { in the empty model }-\hat{\sigma}_{0}^{2} \text { in the model including the characteristic }}{\hat{\sigma}_{0}^{2} \text { in the empty model }} \\
& \mathrm{PCV}_{\mathrm{WE}}=\frac{\hat{\sigma}_{\varepsilon}^{2} \text { in the empty model }-\hat{\sigma}_{\varepsilon}^{2} \text { in the model including the characteristic }}{\hat{\sigma}_{\varepsilon}^{2} \text { in the empty model }}
\end{aligned}
$$

Table 1. Shows three equations of multilevel models under investigation

\begin{tabular}{lll}
\hline Model & Statistical equation combining both levels & Notes \\
\hline 1 & $D R_{i j}=\beta_{0}+u_{0 j}+\varepsilon_{i j}$ & Empty model \\
2 & $D R_{i j}=\beta_{0}+\beta_{1} t i m e_{i j}+u_{0 j}+\varepsilon_{i j}$ & Adding the time variable as fixed-effect \\
3 & $D R_{i j}=\beta_{0}+\beta_{1} t i m e_{i j}+u_{0 j}+u_{1 j} t i m e_{i j}+\varepsilon_{i j}$ & Adding the time variable as fixed- and random-effect \\
\hline
\end{tabular}




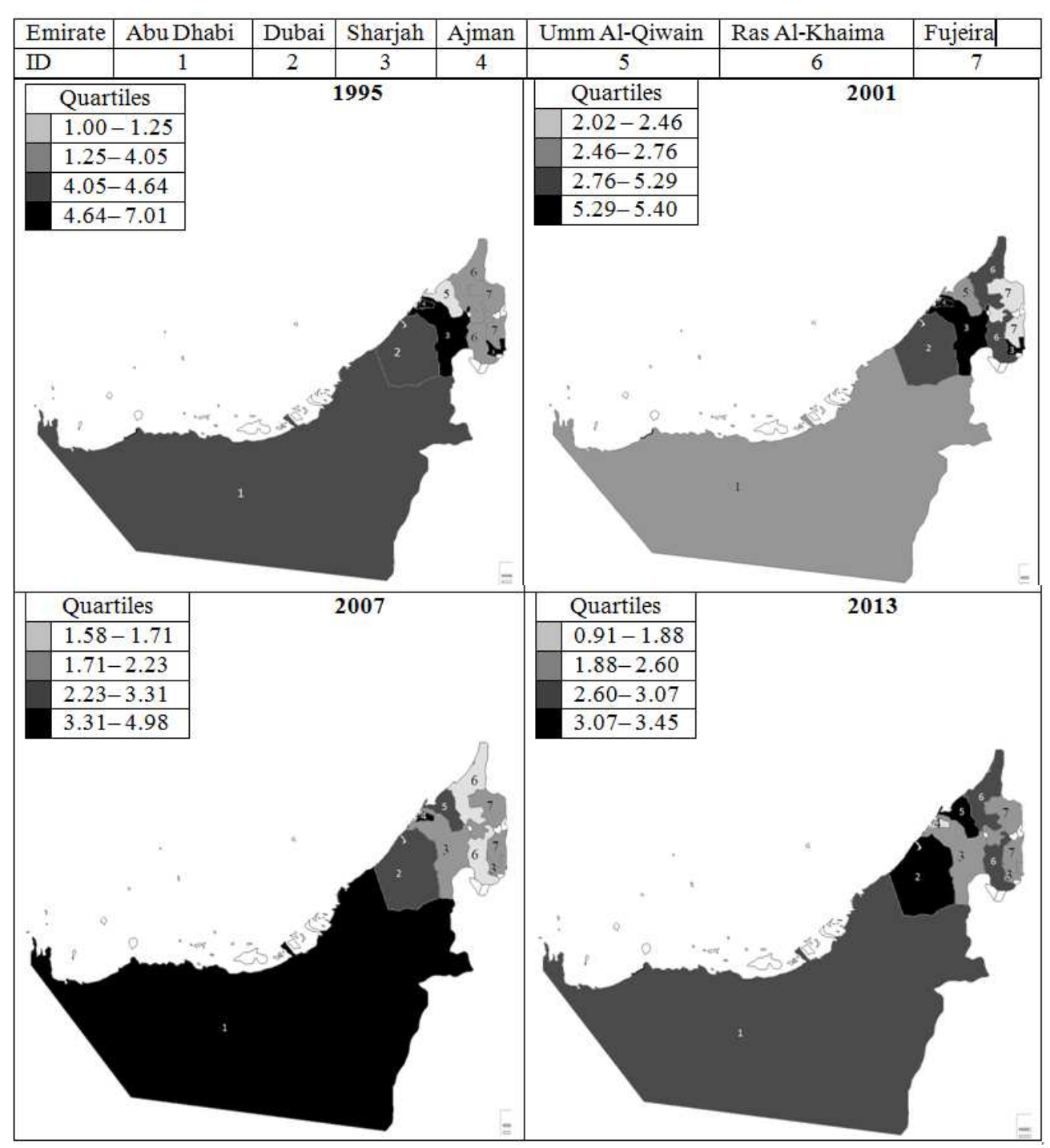

Fig. 2. Shows the maps of the DR for the years 1995, 2001, 2007 and 2013

\section{Spatiotemporal Mapping}

As the saying goes: Picture is better than 1000 words. The DR was investigated for a spatial structure that can be revealed by mapping every 6 years. To construct a choropleth map, the data for 7 emirates were grouped into four classes using quartiles in 4 years: 1995, 2001, 2007 and 2013. A gray tone was assigned for each class. The DR was categorized into four intervals using darker shades of gray to indicate increasing risk in divorce. This approach allows qualitative evaluation of the spatial pattern. Accordingly, maps showing the DR in Fig. 2 explain visual inspection. Based on visual inspection, the DR in 1995 and 2001was almost concentrated in the northern-eastern and middle emirates; the DR in 2007was concentrated in the southern-western emirates and the DR in 2013was concentrated in the northern-western emirates.

\section{Results}

The current study is based on real data and therefore, the results should be used as empirical evidence. The DRs and their descriptive statistics can be shown in Table 2. Across emirates, you can see the highest and lowest mean of the DR were found in Ajman and Fujeira respectively. The highest and lowest Standard Deviation (SD) in the DR were found in Ajman and Ras AlKhaima respectively. The highest and lowest Coefficient of Variation (CV) in the DR were found in Umm AlQiwain and Dubai. Over time, the highest and lowest mean of the DR were found in 2005 and 2012 respectively. The highest and lowest $\mathrm{SD}$ in the DR were found in 1996 and 2012 respectively. The highest and lowest CV in the DR were found in1996 and 2012. Figure 3 shows line chart for the DR of each emirate over the time period (1995-2013) of 19 years. 


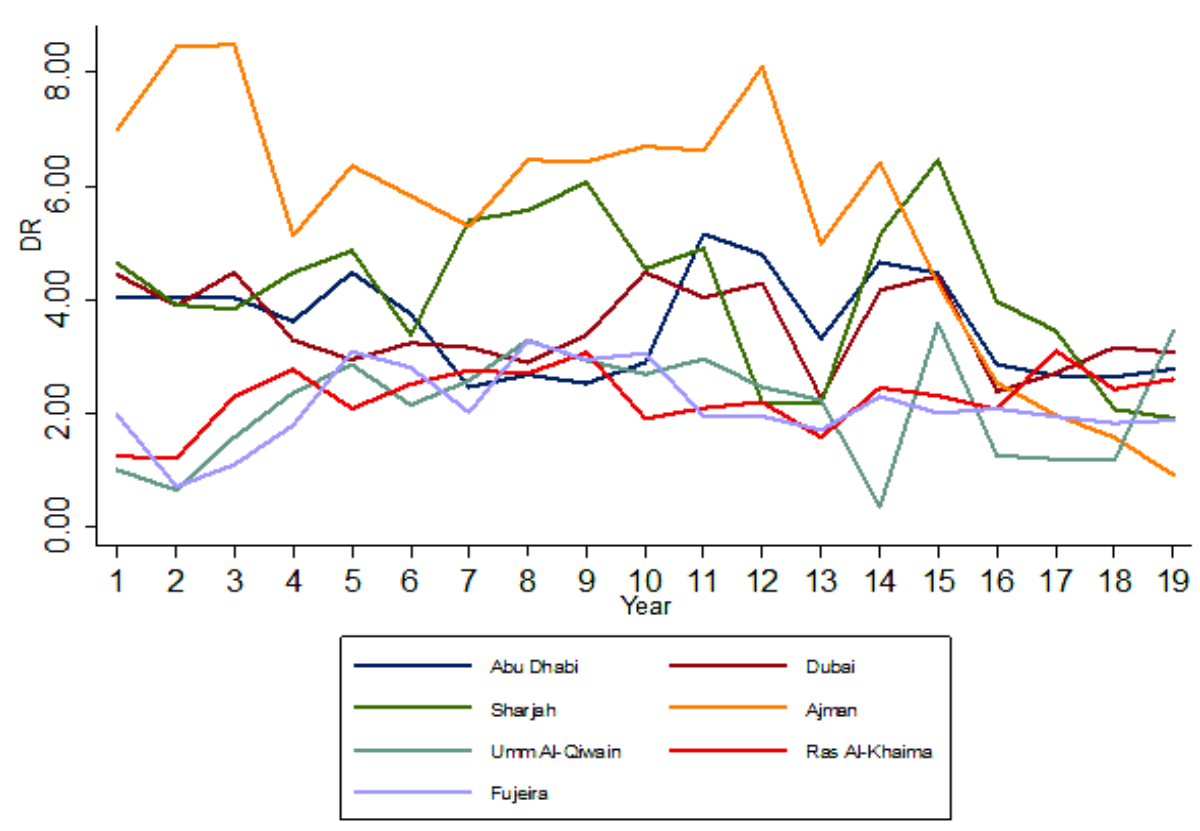

Fig. 3. Shows line chart for the DR of each emirate over the time period (1995-2013) of 19 years

Table 2. Shows the DRs and descriptive statistics over time and across emirates

\begin{tabular}{|c|c|c|c|c|c|c|c|c|c|c|}
\hline Years & Abu Dhabi & Dubai & Sharjah & Ajman & Umm Al-Qiwain & Ras Al-Khaima & Fujeira & Mean & SD & CV (\%) \\
\hline 1995 & 4.05 & 4.45 & 4.64 & 7.01 & 1.00 & 1.25 & 1.96 & 3.48 & 2.18 & 62.65 \\
\hline 1996 & 4.04 & 3.90 & 3.91 & 8.45 & 0.64 & 1.21 & 0.71 & 3.27 & 2.77 & 84.69 \\
\hline 1997 & 4.03 & 4.48 & 3.83 & 8.48 & 1.57 & 2.30 & 1.09 & 3.68 & 2.48 & 67.32 \\
\hline 1998 & 3.62 & 3.28 & 4.47 & 5.13 & 2.36 & 2.76 & 1.80 & 3.35 & 1.17 & 35.04 \\
\hline 1999 & 4.48 & 2.95 & 4.86 & 6.36 & 2.86 & 2.08 & 3.09 & 3.81 & 1.49 & 38.97 \\
\hline 2000 & 3.74 & 3.24 & 3.38 & 5.82 & 2.14 & 2.51 & 2.81 & 3.38 & 1.20 & 35.66 \\
\hline 2001 & 2.46 & 3.16 & 5.40 & 5.29 & 2.57 & 2.76 & 2.02 & 3.38 & 1.38 & 40.97 \\
\hline 2002 & 2.68 & 2.89 & 5.57 & 6.46 & 3.29 & 2.70 & 3.28 & 3.84 & 1.53 & 39.79 \\
\hline 2003 & 2.52 & 3.38 & 6.06 & 6.43 & 2.94 & 3.07 & 2.94 & 3.91 & 1.62 & 41.51 \\
\hline 2004 & 2.89 & 4.48 & 4.55 & 6.70 & 2.69 & 1.90 & 3.06 & 3.75 & 1.61 & 43.04 \\
\hline 2005 & 5.15 & 4.03 & 4.90 & 6.63 & 2.96 & 2.09 & 1.95 & 3.96 & 1.73 & 43.67 \\
\hline 2006 & 4.79 & 4.28 & 2.20 & 8.10 & 2.46 & 2.19 & 1.95 & 3.71 & 2.23 & 60.23 \\
\hline 2007 & 3.31 & 2.27 & 2.18 & 4.98 & 2.23 & 1.58 & 1.71 & 2.61 & 1.19 & 45.48 \\
\hline 2008 & 4.66 & 4.16 & 5.13 & 6.41 & 0.35 & 2.45 & 2.30 & 3.64 & 2.05 & 56.36 \\
\hline 2009 & 4.47 & 4.42 & 6.46 & 4.28 & 3.58 & 2.31 & 2.01 & 3.93 & 1.50 & 38.08 \\
\hline 2010 & 2.86 & 2.39 & 3.98 & 2.54 & 1.26 & 2.07 & 2.08 & 2.45 & 0.84 & 34.15 \\
\hline 2011 & 2.67 & 2.70 & 3.45 & 1.97 & 1.19 & 3.10 & 1.95 & 2.43 & 0.78 & 31.90 \\
\hline 2012 & 2.64 & 3.17 & 2.07 & 1.58 & 1.17 & 2.42 & 1.82 & 2.12 & 0.68 & 31.84 \\
\hline 2013 & 2.78 & 3.07 & 1.92 & 0.91 & 3.45 & 2.60 & 1.88 & 2.37 & 0.86 & 36.22 \\
\hline Mean & 3.57 & 3.51 & 4.16 & 5.45 & 2.14 & 2.28 & 2.13 & & & \\
\hline SD & 0.88 & 0.74 & 1.36 & 2.26 & 0.98 & 0.53 & 0.66 & & & \\
\hline $\mathrm{CV}(\%)$ & 24.69 & 20.98 & 32.75 & 41.57 & 45.72 & 23.38 & 31.21 & & & \\
\hline
\end{tabular}

The $\mathrm{p}<0.01$ of Wald- $\chi^{2}$ was found significant in all models. Given $95 \%$ confidence interval, the fixed and random effects in all models were found significant. When performing statistical modelling, the goodness of fit can be measured by different statistical techniques. One very common technique, which was used in the current study, is the reduction in the deviance. This technique is used to evaluate the fit of consecutive models with additional terms. Table 3 shows that every consecutive model, as compared with the empty model, significantly decreases the deviance and improves the goodness of fit of the model. The deviance is calculated using $-2 \log$ likelihood. As shown from the deviances in Table 3, the multilevel models fit the data much better than the single level time analysis. The deviance cannot be interpreted directly, but rather is compared between models that are fit to the same data set. Suppose that 
two models, model-1 and model-2, have deviances by $\mathrm{D}_{1}$ and $\mathrm{D}_{2}$ with $k_{1}$ and $k_{2}$ parameters respectively. The difference of the deviance $\left(D_{1}-D_{2}\right)$ can be used as a test statistic having a $\chi^{2}$-distribution with $\left(k_{2}-k_{1}\right)$ degrees of freedom (Cho, 2003). For instance, compared to Model-1, Model-2 shows much better fit, due to $\left(D_{1}-D_{2}=10.414\right)$ with $\left(\mathrm{k}_{2}-\mathrm{k}_{1}=1\right)$ and $\mathrm{p}<0.005$. Compared to Model-2, Model-3 shows much better fit, due to $\left(D_{2}-D_{3}=13.738\right)$ with $\left(\mathrm{k}_{3}-\mathrm{k}_{2}=1\right)$ and $\mathrm{p}<0.001$. So, models 2 and 3 show that, as compared with the empty model, every consecutive model significantly decreases the deviance and hence improves the goodness of fit of the model. Also, to search for the best model, the Akaike's Information Criterion (AIC) of the three models were compared, where model-3 has the smallest AIC. It is, therefore, the best model of the three. That's why model-3 was used for the forecasting purpose.

The estimate of fixed-effect of time indicates that the time has a significant influence on the DR. The $\hat{\beta}_{1}=-$ 0.060 , found in models 2 and 3 , can be explained as follows: For a given $j$ th emirate, as the time increases by one year, the DR decreases on average by 0.06 . The fixed effect of intercept, $\hat{\beta}_{0}=3.917$ in models 2 and 3 indicates that, for the DRs within the range of values observed, is the portion of the DR not explained by the time.

In model-3, the total variance, $\hat{\sigma}_{0}^{2}+\hat{\sigma}_{\varepsilon}^{2}$, in the DR that is not explained by the time was found 4.83 . The variance, $\hat{\sigma}_{1}^{2}$ in the DR, that is explained by the time was found $0.8 \%$. However, including the time as a random- effect in model-3 inflates $\hat{\sigma}_{0}^{2}$ in models 1 and 2 from 1.247 to 3.826 . Means that, the between-emirates variance in the DR was inflated due to a composition of several characteristics that presented over time.

Adding explanatory variables in the model could increase the second-level (emirate-level) variance. In cases in which the differences between emirates are hidden by their within-emirate composition, the total variance may decrease as found in the current study, but the emirate component of the variance may increase (Merlo et al. 2005a). Table 3 shows the estimates of fixed- and random-effects, their standard errors and their 95\% confidence intervals. As shown in this table, the $\mathrm{PCV}_{\mathrm{BE}}=-0.48 \%$ of the $\mathrm{DR}$ variance in the empty model-1 was not attributable to the including of fixed-effect of time indicator. The $\mathrm{PCV}_{\mathrm{BE}}$ $=-208.30 \%$ of the DR variance in the empty model- 1 was not attributable to the including of fixed- and random-effects of time indicator. This means [$208.30 \%-(-0.48 \%)=-207.82 \%]$ of the $\mathrm{PCV}_{\mathrm{BE}}$ in the DR in model-1 was not attributable to the including of random-effect of time.

As shown in the same Table 3, the $\mathrm{PCV}_{\mathrm{WE}}=7.99 \%$ of the DR variance in model-1 was attributable to the including of fixed-effect of time indicator. The $\mathrm{PCV}_{\mathrm{WE}}=29.57 \%$ of the DR variance in model-1 was attributable to the including of fixed- and randomeffects of time indicator. This means [29.57-7.99\%= $21.58 \%$ ] of the $\mathrm{PCV}_{\mathrm{WE}}$ in the $\mathrm{DR}$ variance in the empty model-1 was attributable to the including of random-effect of time.

Table 3. Shows the estimates of fixed- and random-effects in three Models (model-1 is an empty, model-2 is a function of fixed-effect of time and model-3 is a function of fixed- and random-effects of time), their standard errors and their $95 \%$ confidence intervals

\begin{tabular}{|c|c|c|c|c|}
\hline \multirow[b]{2}{*}{ Statistic } & & \multicolumn{3}{|c|}{ Estimate (standard error) $(95 \% \mathrm{CI})$} \\
\hline & & Model-1 & Model-2 & Model-3 \\
\hline \multirow[t]{4}{*}{ Fixed } & $\hat{\beta}_{0}$ & $3.311(0.434)$ & $3.917(0.470)$ & $3.917(0.761)$ \\
\hline & & $(2.470,4.169)$ & $(2.996,4.839)$ & $(2.425,5.409)$ \\
\hline & $\hat{\beta}_{1}$ & & $-0.060(0.018)$ & $-0.060(0.038)$ \\
\hline & & & $(-.095,-0.024)$ & $(-.134,0.014)$ \\
\hline \multirow[t]{6}{*}{ Random } & $\hat{\sigma}_{0}^{2}$ & $1.241(0.704)$ & $1.247(0.704)$ & $3.826(2.191)$ \\
\hline & & $(0.409,3.771)$ & $(0.413,3.769)$ & $(1.246,11.753)$ \\
\hline & $\hat{\sigma}_{1}^{2}$ & & & $0.008(0.005)$ \\
\hline & & & & $(0.002,0.030)$ \\
\hline & $\hat{\sigma}_{\varepsilon}^{2}$ & $1.427(0.180)$ & $1.313(0.165)$ & $1.005(0.132)$ \\
\hline & & $(1.114,1.826)$ & $(1.026,1.681)$ & $(0.777,1.301)$ \\
\hline \multirow[t]{2}{*}{ PCV } & Between-emirates & Reference & $-0.48 \%$ & $-208.30 \%$ \\
\hline & Within-emirate (between years) & Reference & $7.99 \%$ & $29.57 \%$ \\
\hline \multirow{2}{*}{\multicolumn{2}{|c|}{ " }} & $0.47(0.145)$ & $0.49(0.145)$ & $0.79(0.098)$ \\
\hline & & $(0.217,0.732)$ & $(0.234,0.747)$ & $(0.542,0.924)$ \\
\hline \multicolumn{2}{|c|}{ Deviance (goodness of fit) 444.744} & 434.33 & 420.592 & \\
\hline \multicolumn{2}{|c|}{ Number of parameters } & 3 & 4 & 5 \\
\hline \multicolumn{2}{|c|}{ AIC } & 450.74 & 442.33 & 430.59 \\
\hline
\end{tabular}


Table 4. Shows the estimated models found from model-3 for each emirate with forecasted values of the DR for the years, 2014 , 2015 and 2016

\begin{tabular}{lllll}
\hline & & Forecasted value & \\
Emirate & Estimated model & -2014 & 2015 & 2016 \\
\hline Abu Dhabi & $D \hat{R}=3.917-.060$ time $+.098+.015$ time & 3.12 & 3.07 & 3.03 \\
Dubai & $D \hat{R}=3.917-.060$ time $+.047+.014$ time & 3.04 & 3.00 & 2.95 \\
Sharjah & $D \hat{R}=3.917-.060$ time $+.977-.015$ time & 3.39 & 3.32 & 3.24 \\
Ajman & $D \hat{R}=3.917-.060$ time $+3.978-.190$ time & 2.90 & 2.65 & 2.40 \\
Umm Al-Qiwain & $D \hat{R}=3.917-.060$ time $-1.735+.058$ time & 2.14 & 2.14 & 2.14 \\
Ras Al-Khaima & $D \hat{R}=3.917-.060$ time $-1.711+.070$ time & 2.41 & 2.42 & 2.43 \\
Fujeira & $D \hat{R}=3.917-.060$ time-1.654+.048time & 2.02 & 2.01 & 2.00 \\
\hline
\end{tabular}

Due to the estimated variance, $\hat{\sigma}_{0}^{2}$, was found significant in all models, there is justification for computing the ICC (Goldstein, 2003). An ICC $\neq 0 \%$ suggests that the emirates are an important determinant of social status, as the observations are nested withinemirates. However, the ICC was found significant in all models based on $95 \%$ CI. This can be considered as a support and confirmation for the role and importance of the within-emirate clustering which was mentioned in the previous studies conducted in other countries. Table 3 shows that about 47,49 and $79 \%$ of the observation residual differences in the DR in models 1,2 and 3 respectively were related to the emirates-level and might be attributable to contextual factors. Alternatively, this clustering might be attributable to the different composition of emirates.

One of the purposes of applying regression analysis models is the forecasting for the response variable given explanatory variable(s). The forecasting can be done for any number of years but the furthest the forecast, the lowest the accuracy can be gotten. So, the values of the DR were forecasted in the years 2014, 2015 and 2016 using model-3 as shown in Table 4 . The DR in most emirates will decrease slightly with a stable rate over the forecasted years. However, many variables are changed, especially on the long-term and accordingly, may affect the change in the DR.

\section{Discussion}

The finding of differences in the DRs are partitioned between the time-level and the emirate-level provides relevant social and epidemiological information. All people living in the same emirate share a common level of socioeconomic and demographic characteristics that differ from the emirate mean in an amount that corresponds to the emirate residual. It is in common sense that observations collected from the same emirate may be more similar to each other in relation to their at least one characteristic than to observations from other emirates. However, persons with similar characteristics may have different degrees of social problems according to whether they live in one emirate or other due to differing of many circumstances such as economic, political, climatic, etc. These contextual indicators express themselves as clustering in some way of individual information within-emirates (Merlo et al., 2005b). That is, a quota if the divorce differences between years under the present study may be attributable to the emirates in which they collect. Some part of the total differences in the DRs between years might be as a consequence of the differences between the emirates. So, one of the objectives of the current study is to answer the question of which part of the total differences in the DRs is greater? The part of variation within-emirates or between-emirates it selves? Based on models 1 and 2, both parts of variation were found approximately same. Based on model-3, the variation between-emirates was found more than three folds compared with the variation within-emirates.

Statistically, it is necessary to use LMM that considers the dependence of the outcome variable between the observations from the same emirate. If this assumption is violated, the results of the regression analysis are biased. However, the clustering in the DRs over time within-emirates is not a statistical nuisance that only needs to be considered for obtaining correct statistical estimations (Merlo et al., 2005b) but a key concept in social and epidemiological fields that yields important information by itself (Merlo et al., 2001; Petronis and Anthony, 2003). Merlo et al. (2005a) stated that the more the people characteristics within a neighborhood are alike, as compared with people in other neighborhoods, the more possible it is that the determinants of individual characteristic are directly related to the contextual environment of the neighborhood, and/or that social processes of geographical segregation are taking place-that is, similar types of people choose or are forced to reside in a given neighborhood. Those aspects are of high significance to reduce inequalities in social and demographic fields on certain geographical areas rather than on specific people only. 
Previous studies applied Multilevel Regression Models (MRMs) using individual observations but in the current study, the rates over time were used. Using rates is more accurate due to the population size of the emirate is taken into account. Snijders and Bosker (1999) stated that when the ICC $=$ zero, the suitability of performing a multilevel analysis is questionable. So, this is one of the several reasons that lead us to apply the MRMs, where, in the current study, the ICC results were found more than $46 \%$ on all models. The correlation results between the random intercept and the random slope were found not significant in all models; that's why they were not shown in the Results section.

The present study may help to provide more insights into the importance of statistical measures of clustering that emerge as appropriate for quantifying contextual phenomena. The current study can be extended in some directions. For instance, more covariates and hierarchical levels, such as municipalities, might be used to provide greater depth and insight to the results if the data can be provided. However, this study supports the use of multilevel regression analysis when dealing with variables measured at different hierarchical levels.

\section{Conclusion and Recommendation}

Statistical measures of multilevel variations can effectively quantify contextual effects in different emirates, which is a relevant issue for understanding the divorce inequalities. The conclusions can be summarized in at least seven aspects: First, across emirates, the highest and lowest mean of the DR were found in Ajman and Fujeira respectively and over time, the highest and lowest mean of the DR were found in 2005 and 2012 respectively. Second, the random-effect of time on the DR was found significant. Third, the moderate to large significant results of ICC explain that the total variance in the DRs is accounted for by the clustering within the same emirate. This clustering could be attributable to the same composition of emirates and the emirate-level is very important in understanding the differences in the DRs. Fourth, the between-emirates variance in the DR was found dramatically larger, more than 3 folds, than the within-emirates variance when the time was included as a random-effect. Fifth, examining the time as fixedand random- effects on the DR variance was found matters within-emirates but not between-emirates. Sixth, based on visual inspection, the DR in 1995 and 2001 was almost concentrated in the northern-eastern and middle emirates, the DR in 2007 was concentrated in the southern-western emirates and the DR in 2013was concentrated in the northern-western emirates. Seventh, the DR will decrease slightly over time in most emirates with almost same and stable rate. Finally, the divorce is a serious issue with major implications for children, men, women and society.
Although the present paper could not study all covariates and their relationship to the divorce, it was possible to highlight important issues raised in social, epidemiological and public health analyses. Due to the ICC results were found moderate to large, focusing intervention on emirates may be an efficient strategy for evaluating the relative importance of the emiratelevel and can promote country resources for specific emirate intervention for those social outcomes that are largely determined by the emirate. So, one of the main recommendations is to focus on creating social and awareness programs and services for the married people, especially for those who are going to be divorced, for the purpose of improving their living conditions and their families and consequently their communities. Adopting government and private training strategies corresponding to the needs of social awareness and guidance centers can create social awareness and improvement. Further studies including the necessary need in studying more explanatory variables such as, demographic, socioeconomic, etc. that probably cause the divorce are required. Also, more levels of analysis, such as municipalities, could be suggested to know in which level the problem of divorce is located and needs more detailed investigation. This will save many of country resources and much of time to handle this problem. The role of other specific emirate-level factors merits further research. If we do not prepare the young people for marriage and help couples to stay together, we will be building up trouble for the society and children. Finally, Policies to improve social awareness need to be monitored every time and every place.

\section{Acknowledgment}

The author thanks the editors of this volume and anonymous reviewers for their valuable feedback and suggestions.

\section{Ethics}

This article is original and contains unpublished material. The corresponding author confirms that all of the other authors have read and approved the manuscript and no ethical issues involved.

\section{References}

Bishop, A.J., G.K. Randall, W.A. Bailey and M. Merten, 2015. Experience of parental marital dissolution earlier in life and the disposition to forgive among older violent and non-violent prisoners. J. Relig. Spirituality Ag., 27: 108-124.

DOI: $10.1080 / 15528030.2015 .1018659$ 
Bourne, P.A., A. Hudson-Davis, C. Sharpe-Pryce, J. Clarke and I. Solan et al., 2014. Does marriage explain murders in a society? In what way is divorce a public health concern? Int. J. Emergency Mental Health, 16: 298-307. DOI: $10.4172 / 1522-4821.1000116$

Cho, S.H., 2003. Using multilevel analysis in patient and organizational outcomes research. Nurs. Res., 52: 61-65. DOI: 10.1097/00006199-200301000-00010

Express, 2013. Ten reasons why so many marriages in the UAE collapse. XPRESS-Al Nisr Media FZ LLC, UAE.

Furtado, D., M. Marcén and A. Sevilla, 2013. Does culture affect divorce? Evidence from European immigrants in the United States. Demography, 50: 1013-1038. DOI: 10.1007/s13524-012-0180-2

Goldstein, H., 2003. Multilevel Statistical Models. 3rd Edn., Hodder Arnold, London.

Hox, J.J., 1995. Applied Multilevel Analysis. 1st Edn., TT-Publikaties, Amsterdam, ISBN-10: 9080107336, pp: 118.

Kreager, D.A., R.B. Felson, C. Warner and M.R. Wenger, 2013. Women's education, marital violence and divorce: A social exchange perspective. J. Marriage Family, 75: 565-581. DOI: 10.1111/jomf.12018

Mednick, B.R., 1987. Long-term effects of parental divorce on young adult male crime. J. Youth Adolesc, 16: 31-45. DOI: 10.1007/BF02141545

Merlo, J., B. Chaix, M. Yang, J. Lynch and L. Råstam, 2005a. A brief conceptual tutorial of multilevel analysis in social epidemiology: Linking the statistical concept of clustering to the idea of contextual phenomenon. J. Epidemiol. Community Health, 59: 443-449.

DOI: $10.1136 /$ jech.2004.023473
Merlo, J., M. Yang, B. Chaix, J. Lynch and L. Råstam, 2005b. A brief conceptual tutorial on multilevel analysis in social epidemiology: investigating contextual phenomena in different groups of people. J. Epidemiol. Community Health, 59: 729-736. DOI: $10.1136 /$ jech.2004.023929

Merlo, J., P. Ostergren, K. Broms, A. Bjorck-Linné and H. Liedholm, 2001. Survival after initial hospitalization for heart failure: A multilevel analysis of patients in Swedish Acute Care Hospitals. J. Epidemiol. Community Health, 55: 323-329. DOI: 10.1136/jech.55.5.323

Merol, J., 2003. Multilevel analytical approaches in social epidemiology: Measures of health variation compared with traditional measures of association. J. Epidemiol. Community Health, 57: 550-552. DOI: $10.1136 /$ jech.57.8.550

Petronis, K. and J. Anthony, 2003. Social epidemiology, intra-neighbourhood correlation and generalized estimating equations. J. Epidemiol. Community Health, 57: 914-914. DOI: 10.1136/jech.57.11.914

Snijders, T. and R. Bosker, 1999. Testing a Model Specification, in Multilevel Analysis-an Introduction to Basic and Advanced Multilevel Modeling. 1st Edn., Sage, Thousand Oaks, Canada.

Webster, N., 2015. Social media and money blamed for increasing UAE divorce rate. The National. Abu Dhabi, Abu Dhabi, UAE: Abu Dhabi Media Company. 